\title{
Mutational analysis reveals separable DNA binding and trans-activation of Drosophila STAT92E
}

\author{
Peter Karsten $^{\text {a }}$, Iris Plischke ${ }^{\text {a }}$, Norbert Perrimon ${ }^{\text {b }}$, Martin P. Zeidler ${ }^{\text {a,* }}$ \\ a Department of Molecular Developmental Biology, Max Planck Institute for Biophysical Chemistry, Am Fassberg 11, 37077 Göttingen, Germany \\ b Department of Genetics, Howard Hughes Medical Institute, Harvard Medical School, 77 Avenue Louis Pasteur, Boston, MA 02115, United States
}

Received 27 June 2005; received in revised form 11 July 2005; accepted 11 July 2005

Available online 29 August 2005

\begin{abstract}
In the canonical model of JAK/STAT signalling STAT transcription factors are activated by JAK mediated tyrosine phosphorylation following pathway stimulation by external cytokines. Activated STAT molecules then homo- or heterodimerise before translocating to the nucleus where they bind to DNA sequences within the promoters of pathway target genes. DNA-bound STAT dimers then activate transcription of their targets via interaction with components of the basal transcription machinery. Here we describe a missense mutation in the SH2 domain of the single Drosophila STAT92E homologue which results in an amino-acid substitution conserved in both the canonical SH2 domain and STAT-like molecules previously identified in C. elegans and the mosquito Anopheles gambiae. This mutation leads to nuclear accumulation and constitutive DNA binding of Drosophila STAT92E even in the absence of JAK stimulation. Strikingly, this mutant shows only limited transcriptional activity in tissue culture based assays and functions as a dominant-negative at both the phenotypic and molecular levels in vivo. These features represent aspects of both dominant gain-of-function and dominant-negative activities and imply that the functions of DNA binding can be functionally separated from the role of STAT92E as a transcriptional activator. It is thus possible that an alternative post-translational modification, in addition to tyrosine phosphorylation, may be required to allow STAT to act as a transcriptional activator and suggests the existence of an alternative mechanism by which STAT transcriptional activity may be regulated in vivo.

(C) 2005 Elsevier Inc. All rights reserved.
\end{abstract}

Keywords: JAK; STAT; SH2 domain; DNA binding; Transcriptional activation

\section{Introduction}

The family of Signal Transducer and Activator of Transcription (STAT) proteins represents a central component of the JAK/STAT pathway. The JAK/STAT signal transduction cascade was originally identified as the primary mediator of interferon induced signalling and has since been shown to be required by multiple cytokine and growth factor receptors. While the pathway is required for multiple developmental processes, its central role in the development, proliferation and differentiation of hematopoietic cell lineages is particularly significant. Indeed, the central role for JAK/STAT signalling in hematopoiesis is highlighted by

\footnotetext{
* Corresponding author. Tel.: +1 49551201 1671; fax: +1 49551201 1755.

E-mail address: mzeidle@gwdg.de (M.P. Zeidler).
}

its association with multiple blood cell malignancies including leukaemias, lymphomas and multiple myelomas (reviewed in Refs. [1-4]).

The human genome encodes seven STAT molecules each of which includes an N-terminal protein-protein interaction domain, a region of structurally conserved coiled-coils, a linker domain, a DNA binding region, a Src Homology 2 (SH2) domain and a C-terminal transcriptional activation domain which contains an absolutely conserved tyrosine residue (Fig. 1A; [5]). Numerous studies have led to the development of a canonical model in which activation of a trans-membrane receptor molecule by an extracellular ligand results in the activation of receptor associated JAK tyrosine kinases. The activated JAKs then phosphorylate both themselves and the associated receptor and so generate a docking site to which the normally cytosolically localised STAT molecules can bind via their 


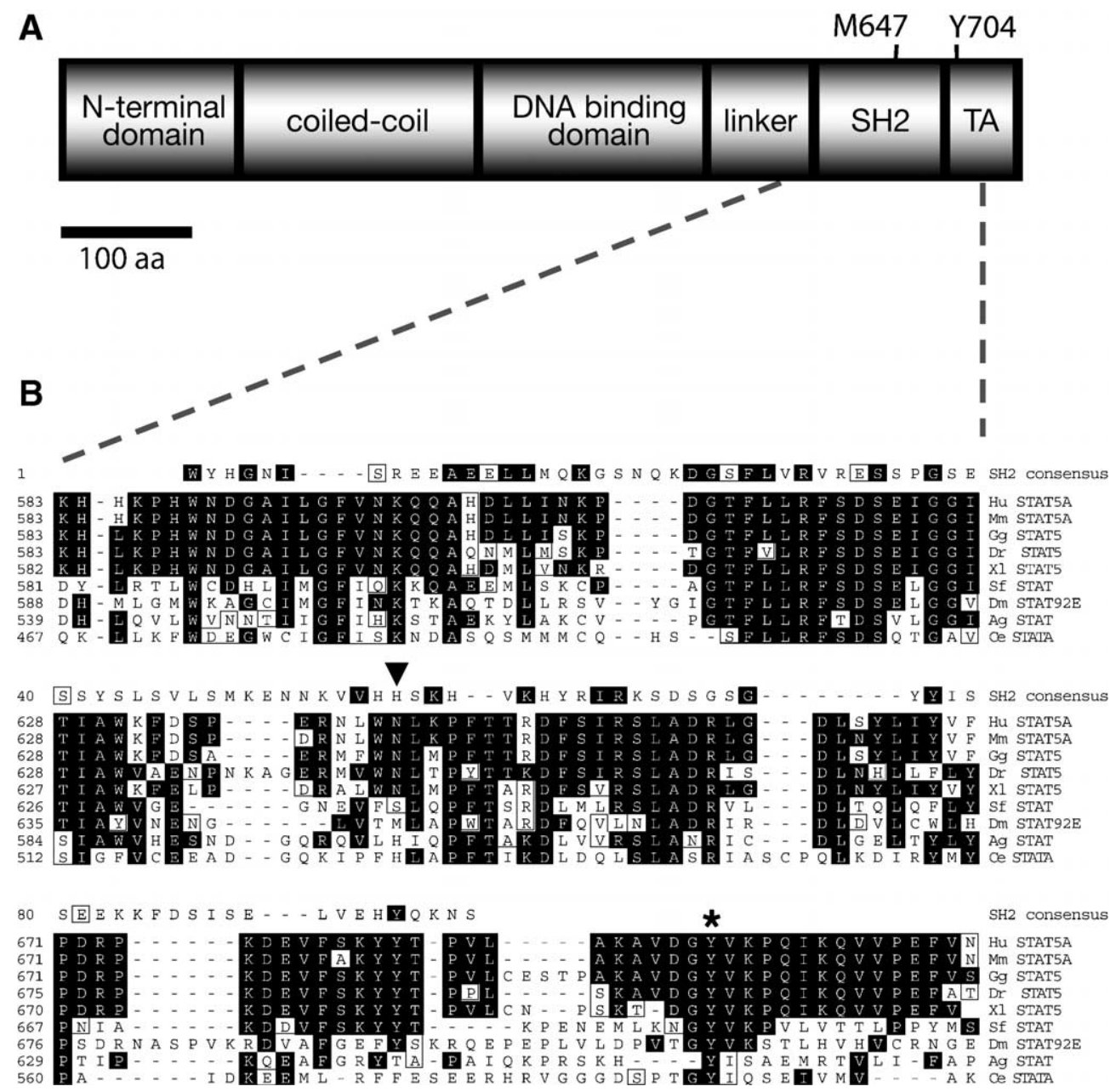

Fig. 1. Alignment and primary structure of STAT92E. (A) Schematic diagram of Drosophila STAT92E showing protein domains and the position of the residues discussed in the text. The relative position of the domains indicated are derived from the alignments shown in Ref. [5]. Scale bar=100 amino acids. $\mathrm{TA}=$ transcriptional activation domain. Dotted lines indicate the region shown in B. (B) Alignment of the SH2 consensus sequence (top line) of a selection of evolutionary conserved STAT-like molecules. Residues aligning to the Drosophila M647 residue are marked by the grey box and the conserved tyrosine residue $\left(\mathrm{Y} 704\right.$ in Drosophila) by an asterisk $\left(^{*}\right) . \mathrm{Hu}=$ Human, $\mathrm{Mm}=$ mouse, $\mathrm{Gg}=$ Chicken, Dr=Zebrafish, $\mathrm{Xl}=$ Xenopus, $\mathrm{Sf}=\mathrm{Moth}, \mathrm{Dm}=$ Drosophila, $\mathrm{Ag}=$ mosquito, $\mathrm{Ce}=C$. elegans .

SH2 domains. The STAT molecules are then themselves phosphorylated on the conserved C-terminal tyrosine residue and form STAT homo- or hetero-dimers. The resulting dimeric STAT complex then translocates into the nucleus where it binds to palindromic DNA binding sites present within the promoters of pathway target genes $[1,2,6]$. Following DNA binding, the transcriptional activation domains of the STAT factors stimulate transcription, presumably via direct or indirect interaction with the basal transcription machinery [7].

Given the central role of JAK/STAT signalling in developmentally important processes it is not surprising that multiple regulatory mechanisms have evolved to control STAT activity. This regulation of STAT activity consists of a balance of both positively and negatively acting factors, a number of which employ post-translational modification to exert their effects. As outlined above, phosphorylation of an invariant tyrosine residue within the $\mathrm{C}$-terminus of all
STATs is a key factor in their activation and is required for the formation of STAT dimers via interactions between their SH2 domains and opposing phospho-tyrosine residues [6]. While several families of tyrosine kinases, including Src and Ableson [8,9], are able to activate the correct tyrosine residue within STATs, by far the most important is the JAK family of receptor associated molecules from which the pathway takes its name [10]. In addition to tyrosine phosphorylation, other post-translational modifications have been identified, which may play roles in STAT regulation, with STAT1 representing a particularly intensively studied example [10]. These modifications include phosphorylation of C-terminal serine residues shown to increase transcriptional activation potential [7], PIAS mediated SUMOlation implicated in the down regulation of transcriptional activity [11], acetylation of a lysine residue within the SH2 domain [12] and a number of contradictory reports regarding the potential methylation of a conserved N-terminal arginine 
residue [13-15]. The presence of these modifications in other STAT molecules, their roles in vivo and their contribution to the overall level of STAT activity are however less clear.

In common with other signalling cascades, the JAK/ STAT pathway has been conserved throughout evolution with STAT-like genes identified in all metazoans as well as the slime mould Dictyostelium [16-18]. The fruit fly Drosophila melanogaster contains the most intensively studied example of an invertebrate JAK/STAT pathway and consists of a single STAT-like gene termed stat92E $[19,20]$ and a single JAK homologue called hopscotch (hop) [21]. The secreted glycoprotein Unpaired (Upd) [22] and two closely related homologues represent pathway ligands, which together with the receptor Domeless [23] make up a functionally 'complete' pathway [18]. Furthermore, negative regulators of the pathway characterised in vertebrate systems are also present and functional in Drosophila and include a family of SOCS proteins [24,25] and a PIAS homologue [26].

In addition to homology between vertebrate and Drosophila JAK/STAT pathway components, a number of the developmental roles for the pathway have also been conserved. These include a role of the pathway in the development and differentiation of Drosophila hematopoietic cells [27,28], a requirement for signalling as a response to bacterial infection [29] and a function in regulating cellular proliferation [30]. In addition, the process of embryonic segmentation, tracheal morphogenesis, foreand hind-gut development as well as the maintenance and development of both the adult testis and ovary have been shown to require pathway signalling (for reviews see Refs. $[16,17,31])$.

Here we describe the generation and characterisation of a point mutation in Drosophila STAT92E. Using both cell based and in vivo assays we show that this mutant is constitutively nuclear translocated and DNA-bound but does not stimulate the normal trans-activation of pathway target genes. This result suggests that the mechanisms by which STAT stimulates transcription are distinct from those required for DNA binding. Given that mutant STAT92E contains all domains present in the wild type protein our findings suggest that DNA binding and transcriptional activation are distinct and may represent separately regulated processes.

\section{Experimental procedures}

\subsection{Cloning}

Fusion proteins, termed STAT92E-GFP, were generated as a tool with which to visualise the activity of pathway signalling on the basis of sub-cellular localisation. The fusion protein consists of the open reading frame of full length STAT92E (splice form CG4257-RC as defined at http://fly.ebi.ac.uk:7081/) amplified with the primers GAGGTACCGAGCATGAGCTTGTGGAAGCGC and ACGGATCCGAAAAGTTCTCAAAGTTTGTAATC from STAT92E cDNA [20], trimmed with Asp718 and BamHI and cloned into pBS-EGFPB. pBS-EGFPB is a cassette based cloning system designed to generate $\mathrm{C}$-terminal fusions with EGFP (Clontech) and was generated by amplifying EGFP with the CAGGATCCGATGGTGAGCAAGGGCGAGGAGCTGTTC and GTTCTAGATTACTTGTACAGCTCGTCCATGCCGAGAGTG primers, which were then trimmed with BamHI and $\mathrm{XbaI}$ before cloning into a similarly cut pBS (KS+) (Stratagene). The resulting EGFP coding region includes a stop codon and the in frame cloning of STAT92E into pBS-EGFPB generates the amino acids $\mathrm{N}$ and $\mathrm{P}$ not originally present in either protein. Point mutations in pBS-STAT92E-GFP were generated using the QuickChange in vitro mutagenesis technique (Strategene) and the primers: CGAAAATGGACTGGTCACCCACCTAGCGCCATGGACTGC and GCAGTCCATGGCGCTAGGTGGGTGACCAGTCCATTTTCG (for $\mathrm{M} 647 \mathrm{H}$ ) and CTAGATCCTGTGACCGGTTTCGTGAAGAGCACATTACATG and CATGTAATGTGCTCTTCACGAAACCGGTCACAGGATCTAG (for Y704F)

Double mutations were generated by two successive rounds of mutagenesis. All constructs were sequenced before subcloning into the inducible Drosophila germline transformation vector $p U A S T$ [32].

The 2xDrafSTATwt reporter has been previously described [33] and was a kind gift of Mi-Ae Yoo. The pAct-Renilla transformation control vector and pAct-Gal4 vectors were generated by cutting the Renilla luciferase and Gal4 coding regions from pRL-SV40 (Promega) and pGAT$\mathrm{B}$, [32] respectively, and subcloning these into the constitutively expressed pAc5.1 (Invitrogen) vector.

\subsection{Genetics}

Drosophila were raised on standard cornmeal/agar food at $25{ }^{\circ} \mathrm{C}$ and standard balancer chromosomes were used as described in http://fly.ebi.ac.uk:7081/. Transgenic lines were generated by microinjection into $w^{1118}$ flies as described in Ref. [34]. Drosophila stocks used include: eyeless-Gal4, which drives expressing throughout the developing eye imaginal disc [35] and nullo-Gal4, which drives high level expression during early embryogenesis (W. Gehring unpublished).

\subsection{Tissue culture}

Drosophila S2 cells [36] were maintained in Schneiders medium $+10 \%$ FCS + Penicillin-Streptomycin (Sigma, $1: 100$ ) at $25{ }^{\circ} \mathrm{C}$ without supplemental $\mathrm{CO}_{2}$. Cells for transfection were split and seeded at approximately $20 \%$ confluency 6-12 $\mathrm{h}$ before transfection using Effectene (QIAGEN). 


\subsection{EMSA}

For EMSAs, cells were lysed in $10 \mathrm{mM}$ HEPES pH 7.9, $10 \mathrm{mM} \mathrm{KCl}, 5 \mathrm{mM} \mathrm{MgCl} 2,1 \%$ TritonX-100, 50\% Glycerol, $1 \mathrm{mM}$ DTT, $1 \mathrm{mM} \mathrm{Na-O}$-Vanadate, $1 \mathrm{mM}$ PMSF 3 days after transfection and incubated $5 \mathrm{~min}$ on ice. Following centrifugation to remove cell debris, supernatant was used for EMSA and Western analysis to normalise for transfection efficiency. Top and bottom oligonucleotides GGATTTTTCCCGGAAATG and GACCATTTCCGGGAAAAA containing the consensus STAT92E binding sequence [19] and top and bottom oligonucleotides GGATTTTTGCCGCAAATG and GACCATTTGCGGCAAAAA containing a mutated STAT92E binding sequence were annealed. The resulting overhangs were then filled by Klenow with dNTPs containing either ${ }^{32} \mathrm{P}-\mathrm{dCTP}$ for radio-labeled or unmodified dCTP for unlabeled oligonucleotides. Binding buffer (20 mM HEPES pH 7.9, $1.4 \mathrm{mM} \mathrm{MgCl} 2,40 \mathrm{mM} \mathrm{KCl}, 0.1 \mathrm{mM}$ EGTA, 5\% Glycerol, $0.5 \mathrm{mM}$ DTT, $1 \mathrm{mM}$ PMSF, $50 \mu \mathrm{g} / \mathrm{ml}$ Poly-dI-dC), $0.1 \mathrm{ng}$ probe and approximately $10 \mu \mathrm{g}$ protein extract were allowed to bind for $30 \mathrm{~min}$ at room temperature. For supershift experiments $\alpha$-GFP antibody (Abcam) was added $(1: 3000)$ to the binding reaction. Complexes were resolved by native polyacrylamide gel electophoresis in a $5 \%$ gel, $0.5 \mathrm{x}$ TBE before drying and autoradiography.

\subsection{Luciferase assays}

Luciferase assays were undertaken in 48 well plates seeded with $6 \times 10^{4} \mathrm{~S} 2$ cells and transfected with $15 \mathrm{ng}$ of pAct-Gal4, $15 \mathrm{ng}$ of the indicated pathway components expressed from the $p U A S T$ transformation vector constructs, $30 \mathrm{ng}$ of 2xDrafSTATwt reporter [33] and $10 \mathrm{ng}$ of pAct-Renilla. pUAST empty vector was added to each transfection as required to maintain a constant concentration of UAS promoters. A 4-day time point was determined to produce maximal activation of the 2xDrafSTATwt reporter while controls containing mutant STAT92E binding sites [33] showed no activation at the same time point (not shown). Cells were lysed with passive lysis buffer (Promega) and measured for firefly and Renilla luciferase activities using the StopAndGlow dual luciferase assay (Promega) and a VictorTM Light 1420 luminescence counter. Transfection efficiency was controlled by Renilla luciferase activity values are normalised to cells transfected with only pAct-Renilla and 2xDrafSTATwt.

\subsection{Histology}

For immuno staining and sub-cellular localisation experiments, S2 cells were seeded on glass coverslips, transfected, and fixed in $\mathrm{PBS}+5 \%$ formaldehyde 3-4 days after transfection. $\alpha$-phospho-STAT92E (Cell Signaling Technologies) was used at 1:500, Сy3 conjugated secondary antibody (Jackson Immuno Research Labs) was used at $1: 200$. Images were captured on a Zeiss Axioskop using Axiocam and Openlab software (Improvision) and on a Leica TCS SP2 confocal microscope using sequential scans. As high levels of transfection were observed to produce false positive staining all cells shown were selected for low and approximately equal transfection as judged by the level of GFP fluorescence.

\subsection{In situ hybridisation}

In situ hybridisation in Drosophila embryos used both sense and anti-sense RNA probes synthesised using DIG RNA labelling kit (Roche) from a trh cDNA (gift of R. Schuh). In situ staining was performed as described by Ref. [37] and photographed using a Zeiss Axioskop, Axiocam and Openlab software (Improvision).

\section{Results}

In an attempt to generate a constitutively active allele of the single Drosophila STAT homologue stat $92 E$ we set out to generate a point mutation based on previous reports identifying constitutively active STAT5A alleles [38]. In tissue culture based screens an asparagine 642 to histidine substitution $(\mathrm{N} 642 \mathrm{H})$ in STAT5A was identified as rescuing growth of an IL-3-dependent $\mathrm{Ba} / \mathrm{F} 3$ cell line in the absence of external cytokine. Protein alignments to other STATs showed that the corresponding amino acid in Drosophila STAT92E is a methionine at position 647 (M647) (Fig. 1B). Strikingly, a number of other STATs present in the mosquito Anopheles gambiae and the nematode $C$. elegans already contain a histidine residue at this position as does a 'consensus' $\mathrm{SH} 2$ domain derived from the alignment of $606 \mathrm{SH} 2$ domains recovered from the ProDom database (Fig. 1B). Given the activating nature of the human STAT5 $\mathrm{A}^{\mathrm{N} 642 \mathrm{H}}$ mutation we introduced an analogous histidine substitution into STAT92E-GFP, a mutation henceforth referred to as STAT92 $\mathrm{E}^{\mathrm{M} 647 \mathrm{H}}$-GFP. The wild type STAT92E-GFP molecule consists of Drosophila STAT92E fused at its C-terminus to EGFP (Clontech). This fusion protein was generated to visualise the sub-cellular localisation of STAT92E and so serve as an in vivo reporter for pathway activity. In addition to wild type STAT92E-GFP and STAT92 $\mathrm{E}^{\mathrm{M} 647 \mathrm{H}}$-GFP, the conserved target of JAK-mediated phosphorylation (Y704; [39]) was mutated to phenylalanine to generate both STAT92 $\mathrm{E}^{\mathrm{Y} 704 \mathrm{~F}}$-GFP and STAT92 $\mathrm{E}^{\mathrm{M} 647 \mathrm{H}, \mathrm{Y} 704 \mathrm{~F}}$-GFP double mutants.

Using tissue culture cells as a model, we first transfected STAT92E-GFP into Drosophila S2 cells [36]. Microscopic examination of transfected cells showed that the sub-cellular localisation of fluorescence appears to at least partially reflect the putative activation state of STAT92E, a finding consistent with activation-dependent 


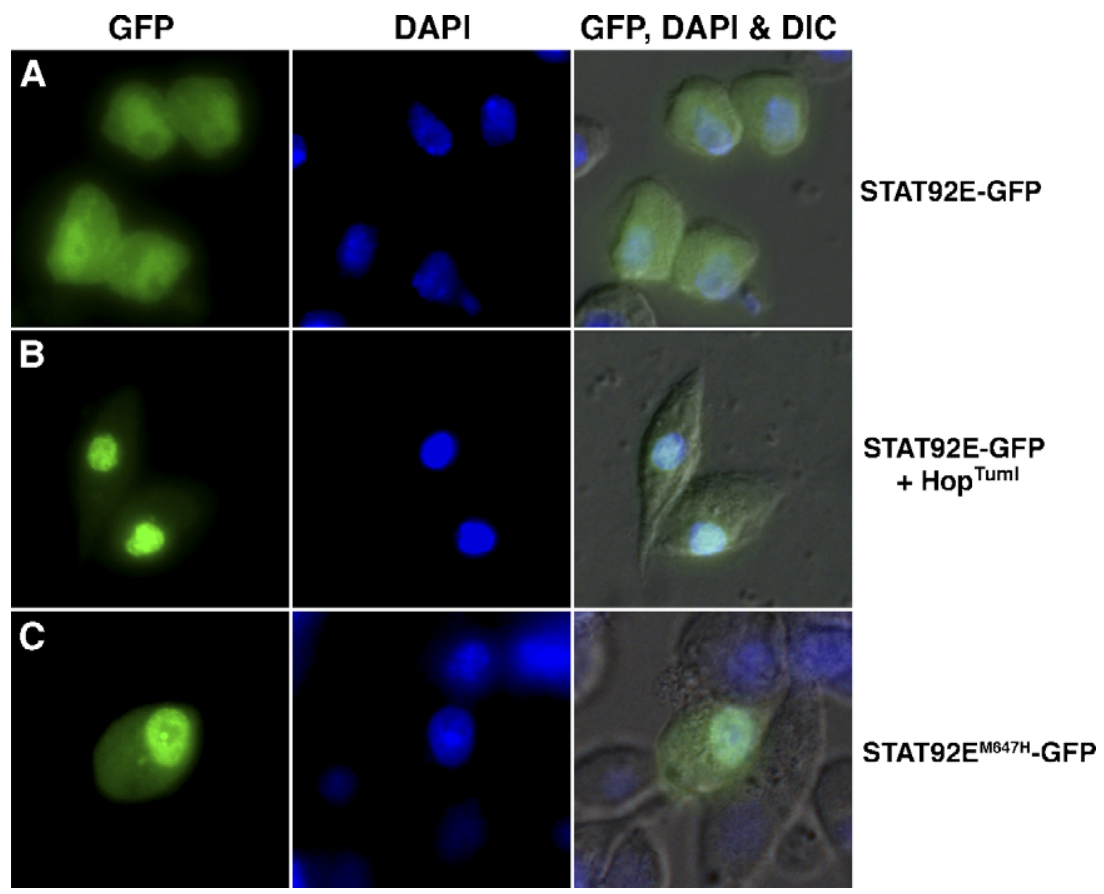

Fig. 2. Sub-cellular localisation of STAT92E-GFP variants. Sub-cellular localisation of STAT92E mutants (A-C). Expression of the indicated STAT92E-GFP fusion proteins in S2 cells shows that GFP is detected in the nucleus of cells containing activated STAT92E. Column 1 is GFP alone, column 2 is DAPI staining of nuclei and column 3 shows GFP, DAPI overlaid on a DIC image of the cells.

nuclear localisation of a number of vertebrate STATs [40]. While wild type STAT92E-GFP is distributed throughout the cell (Fig. 2A), a distinct nuclear enrichment is observed following stimulation by co-transfection with the constitutively active JAK allele Hop ${ }^{\text {Tuml }}$ [41] (Fig.
2B). Strikingly, expression of STAT92 $\mathrm{E}^{\mathrm{M} 647 \mathrm{H}}-\mathrm{GFP}$ results in fluorescence that appears to be nuclear enriched even in the absence of co-expressed Hop ${ }^{\text {Tuml }}$ (Fig. 2C). The mutation $\mathrm{Y} 704 \mathrm{~F}$ is sufficient to prevent the nuclear accumulation of both wild type and STAT92 $\mathrm{E}^{\mathrm{M} 647 \mathrm{H}}-\mathrm{GFP}$
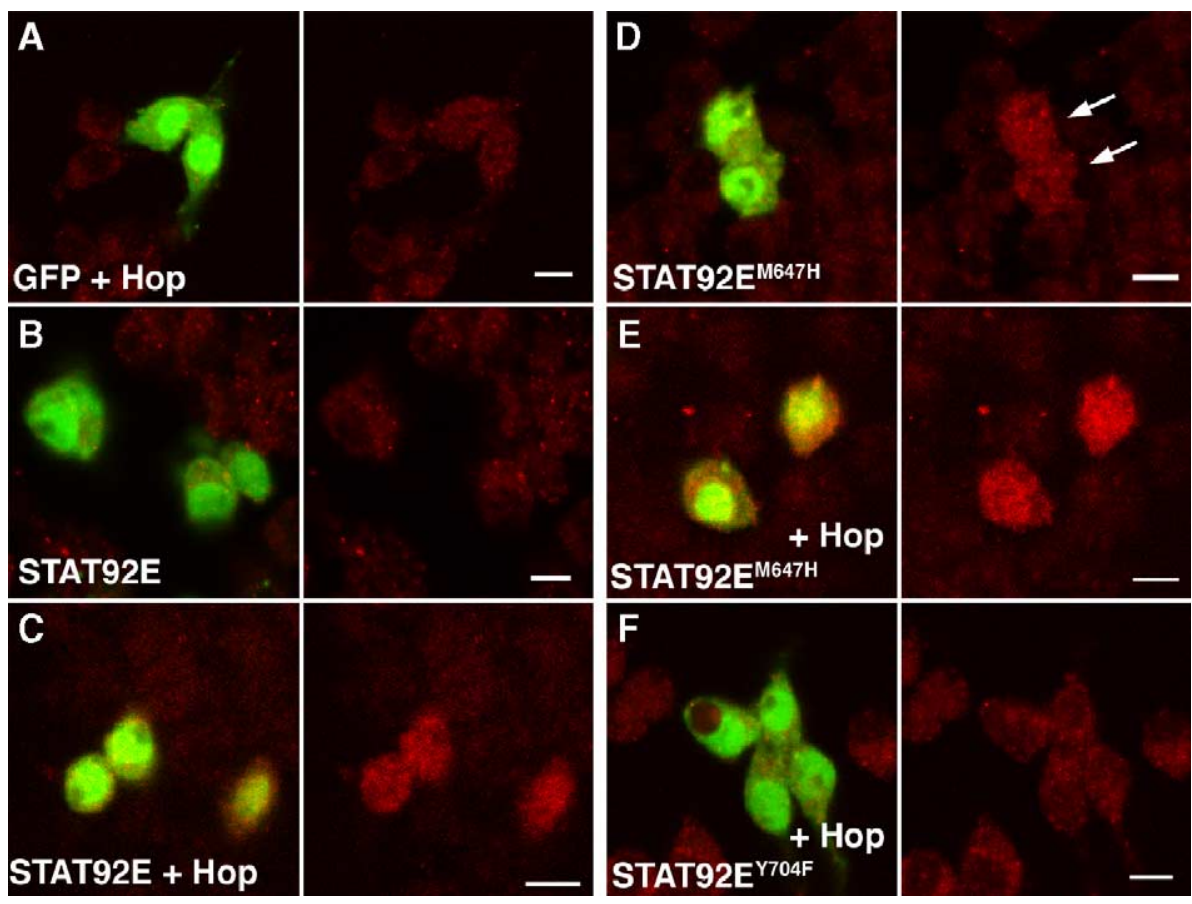

Fig. 3. Phosphorylation state of STAT92E variants. Phosphorylation of STAT92E mutants in S2 cells (A-F). Cells expressing the indicated STAT92E-GFP fusion proteins are visualised by GFP expression (green) and anti-pSTAT92E (red). Left hand panels show the overlay while right hand panels show antipSTAT92E alone. Arrows in G indicate increased levels of pSTAT92E in the absence of Hop. 
molecules even in the presence of co-transfected Hop ${ }^{\text {Tuml }}$ (not shown).

While nuclear localisation is indicative of STAT activity, the phosphorylation of the conserved C-terminal tyrosine residue (Fig. 1A) is essential for the formation of a STAT dimer complex capable of binding to DNA. We therefore set out to investigate the phosphorylation state of the conserved Y704 in wild type STAT92E-GFP and the STAT92 $\mathrm{E}^{\mathrm{M} 647 \mathrm{H}}$ GFP mutation using a STAT92E phospho-specific antibody (anti-pSTAT92E; [39]) in transfected Drosophila S2 cells [36]. Although the anti-pSTAT92E antibody shows some background staining, cells expressing only GFP and Hop appear to contain background, or only slightly raised, levels of detectable pSTAT92E (Fig. 3A). As expected for an antibody displaying phosphorylation-specific binding activity, STAT92E-GFP transfected alone does not increase antipSTAT92E staining (Fig. 3B), whereas STAT92E-GFP coexpressed with Hop results in a clear signal (Fig. 3C). Strikingly, expression of STAT92 $\mathrm{E}^{\mathrm{M} 647 \mathrm{H}}-\mathrm{GFP}$ alone also gives an increase in pSTAT92E (Fig. 3D), and co-expression with Hop results in an even stronger signal (Fig. 3E). As expected, STAT92E-GFP mutants containing the Y704F substitution do not show detectable anti-pSTAT92E staining (Fig. 3F and not shown).

Having shown that STAT92 $\mathrm{E}^{\mathrm{M} 647 \mathrm{H}}$-GFP is constitutively nuclear enriched and appears to be constitutively tyrosine phosphorylated in cells we went on to assess the ability of the STAT92E-GFP mutants to bind to their consensus DNA site [19] using electro-mobility shift assays (EMSA). This approach allows the presence of STAT92E dimers competent to bind DNA in vitro to be detected with high sensitivity. Mock-transfection or transfection of a Hopexpressing plasmid alone resulted in no binding activity indicating that the S2 cells used do not endogenously express levels of STAT92E detectable by this assay. Furthermore, expression of STAT92E-GFP alone shows no binding activity indicating that the upstream components of the pathway are either not present or not activated to a detectable extent (Fig. 4). However, wild type STAT92EGFP, when co-expressed with Hop, produces a single band

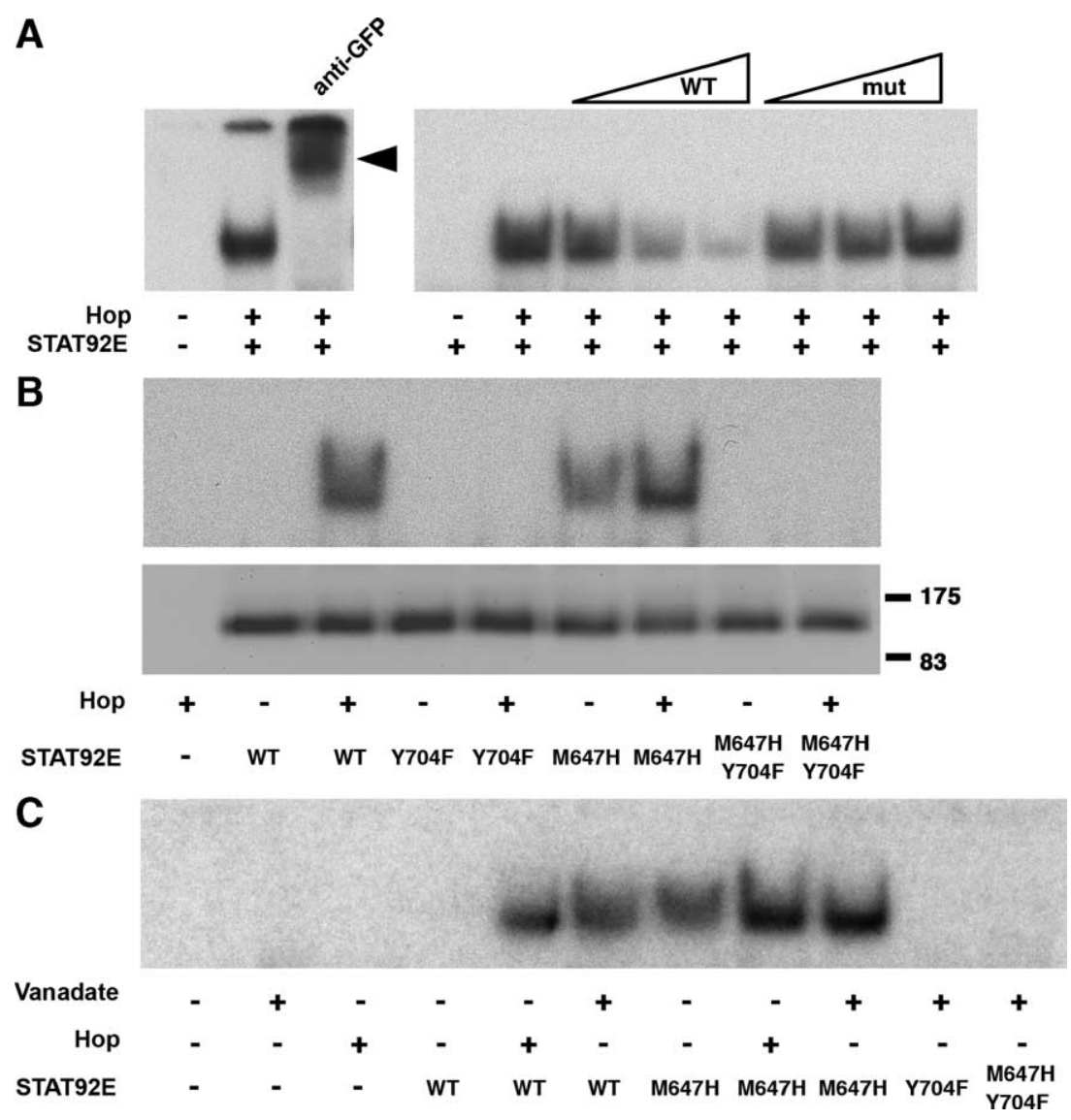

Fig. 4. DNA binding abilities of STAT92E variants. EMSA analysis of STAT92E variants. (A) Wild type STAT92E-GFP binds to a consensus DNA binding site to give a single shifted band that can be super-shifted by anti-GFP antibody $(1: 3000)$ (arrow head). An unlabeled wild type binding site can compete for binding while an binding site containing a single substitution in the consensus binding sequence does not compete (right). Unlabeled wild type and mutant oligonucleotides were added in $10 \times, 50 \times, 100 \times$ excess. (B) STAT92E-GFP mutants transfected with/without Hop bound to radio-labeled consensus DNA binding site show that STAT92 $\mathrm{E}^{\mathrm{M} 647 \mathrm{H}}$-GFP can bind to DNA in the absence of Hop stimulation. Proteins are stable and equally loaded as shown by anti-GFP Western (lower panel). (C) DNA binding activities of the indicated STAT92E proteins following stimulation by Hop or treatment with Sodium-ortho-vanadate. Wild type STAT92E-GFP can also bind DNA in the absence of Hop following treatment with Sodium-ortho-vanadate (lane 6) and gives a signal similar to that produced by the $\mathrm{M} 647 \mathrm{H}$ mutant in the absence of vanadate (lane 7). 
that can be super-shifted by the addition of anti-GFP antibody (Fig. 4A). Furthermore, this band can be competed by an excess of unlabeled oligonucleotides containing the consensus STAT92E binding site but not by oligonucleotides containing mutations in the binding site (Fig. 4A). Together, these experiments demonstrate that the EMSA technique is capable of specifically detecting STAT92E complexes which show specificity for the previously determined DNA recognition site.

We next examined the protein levels of the STAT92EGFP mutations expressed in S2 cells by Western blotting of whole cell lysates, and determined that all STAT92E-GFP variants are both expressed and stable with no major degradation products detected (Fig. 4B and not shown). Using quantification data from these Western blots, we normalised protein levels before checking for DNA binding activity by EMSA. While wild type STAT92E-GFP is activated only when co-transfected with Hop, the STAT92 $\mathrm{E}^{\mathrm{M} 647 \mathrm{H}}$-GFP mutant yields a clear band, indicating that this protein can constitutively bind to DNA even in the absence of Hop (Fig. 4B, lane 6). Moreover this band migrates at the same position as the band shifted by wild type STAT92E-GFP indicating that STAT92 $\mathrm{E}^{\mathrm{M} 647 \mathrm{H}}$-GFP is likely to bind to DNA as a dimer. Co-expression of Hop increases the strength of the shifted band while Y704F mutants in both wild type and M647H backgrounds show no DNA binding activity, indicating that DNA binding of both STAT92E-GFP alleles is dependent on the integrity of Y704.

Given the apparent requirement for Y704 phosphorylation to produce a dimer capable of binding DNA and the absence of detectable Hop activity in the S2 cell line the question arises regarding the mechanism by which STAT92 $\mathrm{E}^{\mathrm{M} 647 \mathrm{H}}$-GFP may be activated. In an attempt to mimic this Hop independent activation, we repeated previous experiments in which the inhibitor of protein phosphatases Sodium-ortho-vanadate was shown to activate STAT92E [19,42]. Both co-transfection with Hop and treatment with Sodium-ortho-vanadate are sufficient to stimulate wild type STAT92E-GFP DNA binding (Fig. $4 \mathrm{C}$, lanes 5 and 6 ). Given that the bandshifts stimulated by treatment with phosphatase inhibitors are similar to those detected with unstimulated STAT92 $\mathrm{E}^{\mathrm{M} 647 \mathrm{H}}$-GFP, these results suggest that low levels of tyrosine kinase activity are endogenously present in S2 cells and are capable of activating both forms of STAT92E. Furthermore, these results indicate that the activity of endogenous protein phosphatases normally counter this low level non-specific STAT92E stimulation, yet are incapable of exerting a similar negative influence on the STAT92 $\mathrm{E}^{\mathrm{M} 647 \mathrm{H}}$-GFP allele.

When considered in combination, it appears that the STAT92 $\mathrm{E}^{\mathrm{M} 647 \mathrm{H}}$-GFP mutant fulfils the characteristics typical of activated wild type STAT92E-GFP following stimulation by Hop. Furthermore, the apparent activity of STAT92 $\mathrm{E}^{\mathrm{M} 647 \mathrm{H}}$-GFP is dependent on the integrity and phosphorylation of the conserved Y704 residue suggesting that both the tyrosine phosphorylation and protein:protein interactions of STAT92 $\mathrm{E}^{\mathrm{M} 647 \mathrm{H}}$-GFP are reminiscent of normal STAT activation. As such, STAT92 $\mathrm{E}^{\mathrm{M} 647 \mathrm{H}}$-GFP appears to represent a constitutive gain-of-function allele as judged by the three criteria of sub-cellular localisation, DNA binding ability and Y704 phosphorylation.

Although phosphorylation, nuclear translocation and DNA binding are required for STAT activity, the key role for this transcription factor is its ability to elicit the expression of pathway target genes. We therefore determined the ability of the $\mathrm{M} 647 \mathrm{H}$ mutant to activate transcription in Drosophila S2 cells using a luciferase reporter containing two STAT92E binding sites derived from the Draf promoter [33]. Using this reporter in S2 cells, expression of either STAT92E-GFP, Hop or Hop ${ }^{\text {Tuml }}$ alone produces no luciferase activity above background levels. However, co-expression of wild type STAT92E-GFP together with either Hop or Hop ${ }^{\text {Tuml }}$ results in a 6 to 8 fold response (Fig. 5). As expected, a reporter containing mutated STAT92E binding sites shows no response demonstrating that activation is dependent on STAT92E binding ([33] and not shown). Strikingly, expression of the constitutively phosphorylated and DNA-bound STAT92 $\mathrm{E}^{\mathrm{M} 647 \mathrm{H}}$-GFP allele is only capable of increasing reporter gene activity by around $50 \%$, and even coexpression with Hop or Hop ${ }^{\text {Tuml }}$ only results in a relatively modest 2 fold increase in luciferase activity, about $1 / 4$ of the activation obtained by stimulated wild type STAT92E-GFP (Fig. 5). By comparison to wild type STAT92E-GFP it therefore appears that STAT92 $\mathrm{E}^{\mathrm{M} 647 \mathrm{H}}$-GFP is incapable of strongly activating transcription in this tissue culture based assay. As expected from the EMSA results, STAT92E-GFP alleles containing Y704F mutations show no activity (not shown).

Given the apparently contradictory results suggesting constitutive DNA binding and only low levels of transcriptional activation, we moved our analysis in vivo so as to analyze the function of STAT92E mutants in the context of the whole fly. The JAK/STAT pathway is required for a range of developmental processes during embryonic, larval and adult life (for reviews see Refs. [31,43]). One of these requirements is for the proliferation of cells within the eye imaginal disc, the larval tissue that gives rise to the adult compound eye. Loss of JAK/STAT pathway activity results in a small-eye phenotype while ectopic pathway activation is sufficient to stimulate cellular over-proliferation and an overgrown adult eye $[44,45]$. We therefore expressed components of the JAK/STAT pathway throughout the developing eye imaginal disc using the eye disc-specific driver line eyeless-Gal4 (ey-Gal4) [35]. While expression of GFP has no effect on the size or morphology of the resulting adult eye (Fig. 6A-B), mis-expression of a dominantnegative version of the pathway receptor Domeless (Dome $\Delta$ Cyt; [23]) produces a strong reduction in the overall volume of the eye and a loss of ventral tissue (Fig. $6 \mathrm{C}-\mathrm{D})$. By contrast the ectopic pathway activation caused 


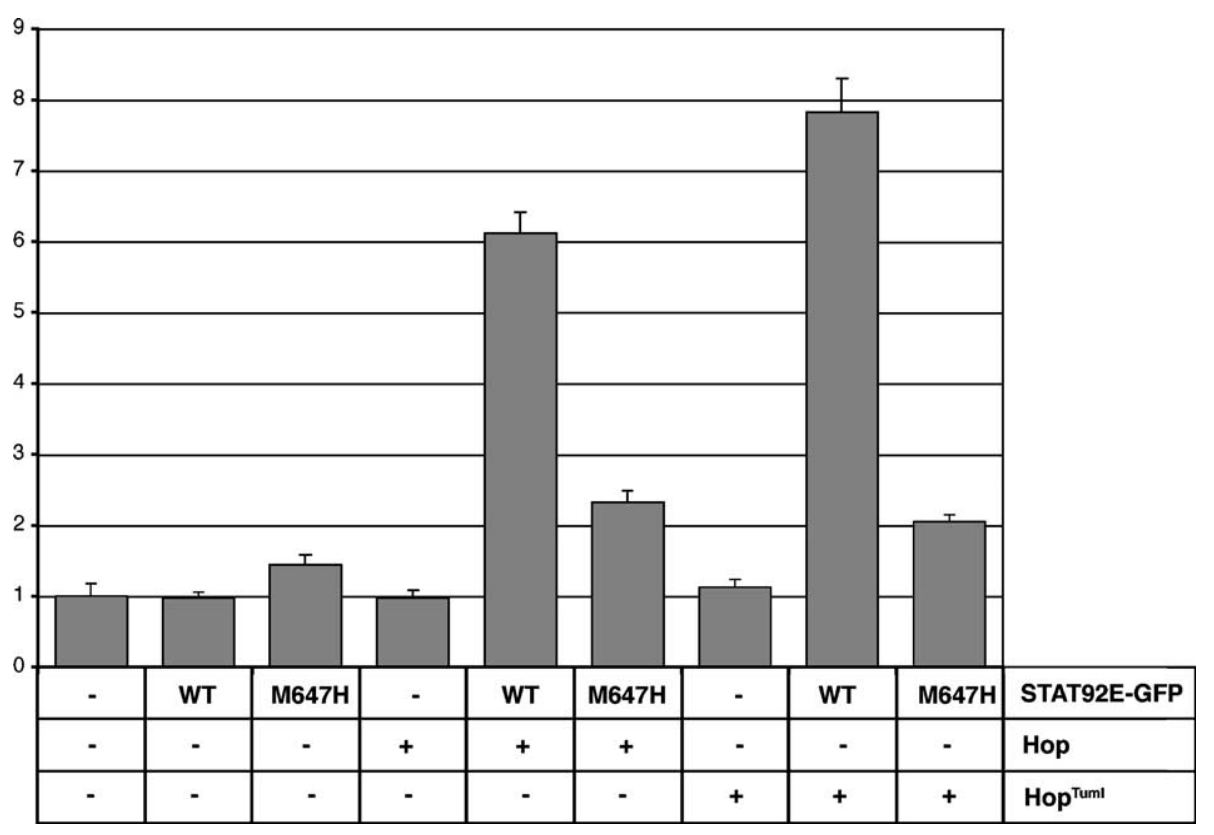

Fig. 5. Transcriptional activation by STAT92E variants. Transcriptional activity of STAT92E mutants. Fold activation of a luciferase reporter construct present in cells transfected with the indicated combination of STAT92E-GFP fusion proteins and Hop/Hop ${ }^{\text {Tuml }}$. Error bars represent standard deviations of one representative experiment undertaken in quadruplicate.

by mis-expression of Hop ${ }^{\text {Tum-1 }}$ causes overgrowth of the adult eye (Fig. 6E-F). We therefore used this assay to assess the function of STAT92E-GFP mutants in the context of eye growth and development. Although expression of wild type STAT92E-GFP results in occasional mild roughening in posterior equatorial regions, no discernable difference in eye size is observed (Fig. $6 \mathrm{G}-\mathrm{H}$ ). By contrast, expression of STAT92 $\mathrm{E}^{\mathrm{M} 647 \mathrm{H}}$-GFP from multiple independent transgenic lines is sufficient to produce roughening, a reduction of eye volume and loss of ventral eye tissue (Fig. 6I-J), an effect analogous to that produced by Dome $\Delta$ Cyt. Mutations containing Y704F substitutions have no dominant effect in otherwise wild type or M647H backgrounds (Fig. 6K-N), indicating that STAT92 $\mathrm{E}^{\mathrm{M} 647 \mathrm{H}}$-GFP requires this residue to exert its dominant-negative effect.

The eye size assays indicate that mis-expression of STAT92 $\mathrm{E}^{\mathrm{M} 647 \mathrm{H}}$-GFP produces developmental defects consistent with its function as a dominant-negative allele in vivo. In order to confirm this effect we extended our analysis to the molecular level by analyzing the expression of the JAK/STAT pathway target gene trachealess (trh) (Fig. 7A; [23]). trh is one of the first markers of tracheal development, and encodes a protein required for the development of the embryonic and larval tracheal system
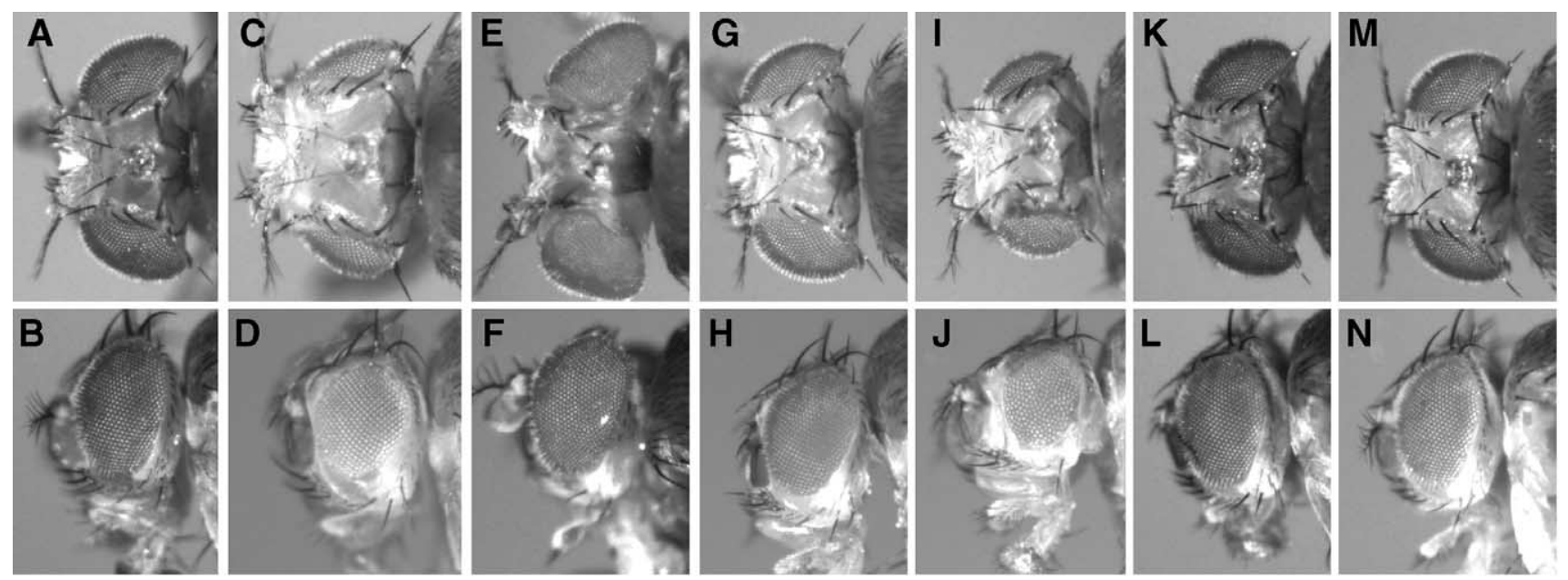

EGFP

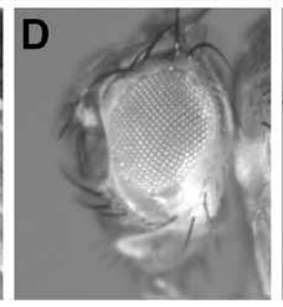

Dome $\Delta$ Cyt

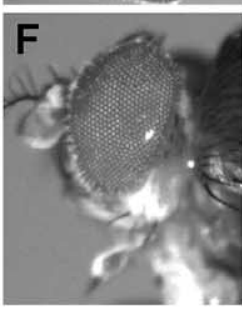

Hop ${ }^{\text {Tuml }}$

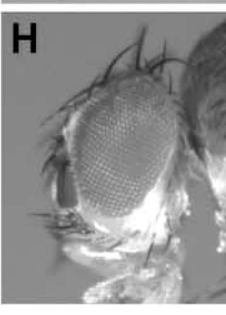

STAT92E

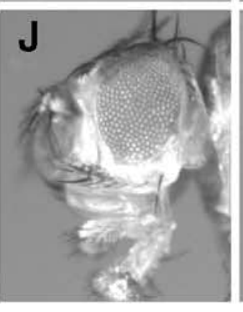

STAT92E ${ }^{\text {M647H }}$
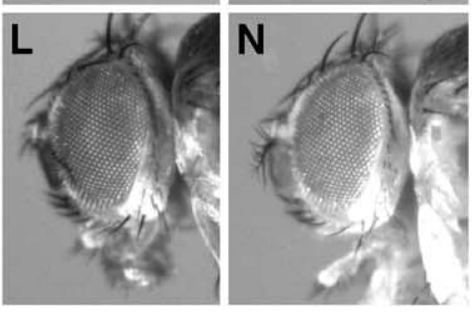

STAT92E ${ }^{\text {Y704F }}$ STAT92E ${ }^{\text {M647H Y704F }}$

Fig. 6. Effect of STAT92E variants mis-expression on the developing eye. (A-N) Adult eyes expressing the indicated controls and STAT92E-GFP fusion proteins under the control of ey-Gal4. All eyes are anterior to the left. Eyes in the upper panel show the dorsal view, eyes in the lower panel the lateral view. A reduction in the size and volume of the adult eye in $\mathrm{C}, \mathrm{D}$ and $\mathrm{I}, \mathrm{J}$ is apparent. 

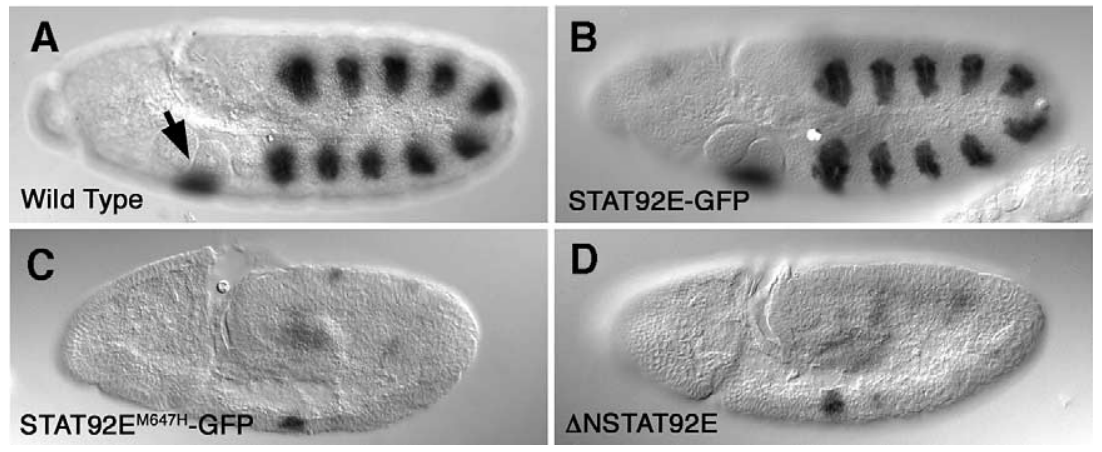

Fig. 7. Expression of the JAK/STAT pathway target gene trh. trh expression in stage 10/11 embryos shown dorsal up and anterior to the left. Wild type (A) and nullo-Gal4/UAS-STAT92E-GFP expressing embryos (B) show strong trh expression localised within the ten tracheal placodes and the presumptive salivary glands (arrow in A). By contrast, expression of trh is almost completely ablated in embryos of the same stage expressing $n u l l o-G a l 4 / U A S-S T A T 92 E^{M 647 H}-G F P$ (C) or nullo-Gal4/UAS- NSTAT92E (D). Note that while expression of trh in the salivary gland does not require STAT92E [23], ubiquitous mis-expression of dominant-negative STAT92E variants is sufficient to prevent expression in this tissue.

[46]. As previous studies have shown that loss of Domeless or STAT92E is sufficient to ablate trh expression in the stage 9 embryo [23] we used the expression of this gene as a molecular marker for pathway activity while simultaneously using the nullo-Gal4 line to ubiquitously drive transgene expression. As expected, nullo-Gal4 driven over-expression of wild type STAT92E-GFP has no effect on trh expression (Fig. 7B). However, despite the presence of endogenous STAT92E activity in the developing tracheal placodes, misexpression of STAT92 $\mathrm{E}^{\mathrm{M} 647 \mathrm{H}}$-GFP is sufficient to strongly reduce the levels of trh expressed, and no tracheal pits form in these segments (Fig. 7C). This effect is very similar to that produced by the mis-expression of the dominantnegative splice form of STAT92E termed $\triangle$ NSTAT92E (Fig. 7D; [47]), and indicates at the molecular level that STAT92 $\mathrm{E}^{\mathrm{M} 647 \mathrm{H}}$-GFP acts as a dominant-negative capable of preventing the function of endogenous wild type STAT92E in vivo.

\section{Discussion}

We have shown that Drosophila STAT92E containing a mutation of methionine 647 to histidine results in a molecule that constitutively nuclear accumulates, is constitutively DNA-bound and is likely to be constitutively tyrosine phosphorylated. However, STAT92 $\mathrm{E}^{\mathrm{M} 647 \mathrm{H}}$-GFP is largely incapable of activating transcription and acts as a dominant-negative in vivo such that its expression is sufficient to inhibit endogenous JAK/STAT pathway activity both at the phenotypic and gene expression levels. Given these findings it appears possible that the dominant-negative effect occurs via target gene promoter occupation by transcriptionally incompetent STAT92 $\mathrm{E}^{\mathrm{M} 647 \mathrm{H}}$-GFP complexes, which block access for endogenous STAT92E.

In the light of our results it is perhaps surprising that the original substitution on which the Drosophila mutation was based has been described as a gain-of-function allele
[38]. In this report, human STAT5 $\mathrm{A}^{\mathrm{N} 643 \mathrm{H}}$ was identified on the basis of its ability to rescue cytokine independent growth of the $\mathrm{Ba} / \mathrm{F} 3$ cell line. While this rescue acted as the initial selection criteria, analysis of known STAT5A targets revealed only relatively modest increases in transcription induced by the activated molecule [38] suggesting that a small increase in the level of endogenous JAK/STAT pathway activity may be sufficient to rescue the $\mathrm{Ba} / \mathrm{F} 3$ cell line. Given that multiple STATs are expressed in $\mathrm{Ba} / \mathrm{F} 3$ cells, such a low level increase in activity could conceivably be mediated by hetero-dimers containing transcriptionally competent endogenous STATs and constitutively phosphorylated STAT5 $\mathrm{A}^{\mathrm{N} 643 \mathrm{H}}$ molecules. By contrast, the low redundancy of the Drosophila system reduces the likelihood that similar heterodimeric combinations form in vivo or in S2 cells, especially given the relatively high levels of expression that result from transient transfection and the Gal4/UAS system [32]. As such, a scenario in which STAT92 $\mathrm{E}^{\mathrm{M} 647 \mathrm{H}}$-GFP is constitutively DNA-bound yet transcriptionally incompetent may represent the more accurate description of this particular mutation in vivo.

Although this study has shown that the DNA binding and transcriptional activation activities of STA92E represent two distinct and separable processes the mechanism by which STAT92 $\mathrm{E}^{\mathrm{M} 647 \mathrm{H}}$-GFP is constitutively phosphorylated in the absence of active Hop is not clear.

As shown above, the inhibition of endogenous phosphatase activity by Sodium-ortho-vanadate treatment is sufficient to stimulate Y704-dependent DNA binding of wild type STAT92E-GFP (Fig. 4C). Given the lack of detectable endogenous JAK/STAT pathway activity in $\mathrm{S} 2$ cells, it appears that tyrosine kinases other than Hop are able to phosphorylate STAT92E. Furthermore, Sodium-ortho-vanadate treatment shows that the action of these non-specific kinases is normally countered by the activity of endogenous phosphatase activity. In the case of STAT92 $\mathrm{E}^{\mathrm{M} 647 \mathrm{H}}$-GFP, however, JAK-independent phosphorylation of Y704 does 
not appear to be countered by endogenous phosphatases. Intriguingly, the possibility of an increased $\mathrm{pY} / \mathrm{SH} 2$ affinity in STAT92 $\mathrm{E}^{\mathrm{M} 647 \mathrm{H}}$-GFP is consistent with molecular modeling, based on the known structures of homo-dimerised STAT3 [6]. Using such an approach, it appears that vertebrate STAT5A $^{\mathrm{N} 643 \mathrm{H}}$ and Drosophila STAT92E $\mathrm{S}^{\mathrm{M} 647 \mathrm{H}}$ mutants both substitute residues with the potential to physically interact with the phospho-tyrosine residue of the dimerised partner (P. Montaville and S. Becker, personal communication; [38]). Whether the difference between wild type and STAT92 $\mathrm{E}^{\mathrm{M} 647 \mathrm{H}}$ is a consequence of biophysical factors such as an increased affinity between $\mathrm{pY}$ and the SH2 domain and/or decreased accessibility for phosphatases remains to be determined.

A second question raised by the activity of STAT92 $\mathrm{E}^{\mathrm{M} 647 \mathrm{H}}$-GFP is the nature of the mechanism by which STAT92E functions as a transcriptional activator. Numerous studies have shown that endogenous STAT92E acts as a transcriptional activator both in cell culture and in vivo and a number of target genes have been identified, which require active pathway signalling for their expression $[20,23,33,48,49]$. Given that STAT92 $\mathrm{E}^{\mathrm{M} 647 \mathrm{H}}$-GFP appears to be constitutively phosphorylated and DNA-bound the reason for its failure to activate transcription is less clear. Indeed, the STAT92 $\mathrm{E}^{\mathrm{M} 647 \mathrm{H}}$-GFP molecule contains all domains present in wild type STAT92E and differs by only a single $\mathrm{SH} 2$ domain internal residue. While it is possible that the $\mathrm{M} 647 \mathrm{H}$ substitution may result in unfolding or instability of a distinct trans-activation domain, this appears to be unlikely given the physical location of the mutated residue and the presence of histidine residues in analogous positions both within other STAT molecules and other SH2 domains shown be active in vivo (Fig. 1B). Rather, it seems that the full transactivation activity of STAT92E is not an inherent characteristic of the molecule itself but is likely to require a second post-translational modification, in addition to tyrosine phosphorylation, that is missing in STAT92 $\mathrm{E}^{\mathrm{M} 647 \mathrm{H}}$-GFP.

Finally, the presence of endogenous histidine residues in mosquito and $C$. elegans STATs at the position mutated in Drosophila STAT92E ${ }^{\mathrm{M} 647 \mathrm{H}}$-GFP (Fig. 1B) also raises the possibility that STAT-like molecules present in these organisms may be constitutively DNA-bound. Furthermore, despite the availability of genomic sequence data no JAKlike molecule has been identified in $C$. elegans. While inherently speculative, it is possible that STAT activity in $C$. elegans may be controlled by mechanisms independent of tyrosine phosphorylation, conceivably via the modulation of transcriptional activation activity.

\section{Acknowledgements}

We wish to thank Mi-Ac Yoo, S. Newfeld, H. Sun, R. Schuh, G. Vorbrüggen, M. Henriksen and W. Gehring for fly stocks and reagents. S. Häder provided valuable technical assistance. We thank P. Montaville and S. Becker for valuable insights into structural models. This work was supported by an Emmy Noether Fellowship of the Deutsche Forschungsgemeinschaft (DFG) and the Max Planck Society. Initial development of STAT92E-GFP was undertaken in the NP lab and was supported by an EMBO Long Term Postdoctoral fellowship to MPZ.

\section{References}

[1] T. Kisseleva, S. Bhattacharya, J. Braunstein, C.W. Schindler, Gene $285(1-2)(2002) 1$.

[2] J. Bromberg, J.E. Darnell Jr., Oncogene 19 (21) (2000) 2468.

[3] J.E. Darnell Jr., I.M. Kerr, G.R. Stark, Science 264 (5164) (1994) 1415.

[4] D.E. Levy, J.E. Darnell Jr., Nat. Rev. Mol. Cell Biol. 3 (9) (2002) 651.

[5] M.H. Heim, in: P.B. Sehgal, D.E. Levy, T. Hirano (Eds.), Signal Transducers and Activators of Transcription (STATs); Activation and Biology, Kluwer Academic Publishers, p. 11.

[6] S. Becker, B. Groner, C.W. Muller, Nature 394 (6689) (1998) 145.

[7] D.E. Levy, in: P.B. Sehgal, D.E. Levy, T. Hirano (Eds.), Signal Transducers and Activators of Transcription (STATs); Activation and Biology, Kluwer Academic Publishers, p. 327.

[8] C.L. Yu, D.J. Meyer, G.S. Campbell, A.C. Larner, C. Carter-Su, J. Schwartz, R. Jove, Science 269 (5220) (1995) 81.

[9] M. David, E. Petricoin 3rd, C. Benjamin, R. Pine, M.J. Weber, A.C. Larner, Science 269 (5231) (1995) 1721.

[10] T. Decker, M. Muller, P. Kovarik, in: P.B. Sehgal, D.E. Levy, T. Hirano (Eds.), Signal Transducers and Activators of Transcription (STATs); Activation and Biology, Kluwer Academic Publishers, p. 207.

[11] D. Ungureanu, S. Vanhatupa, N. Kotaja, J. Yang, S. Aittomaki, O.A. Janne, J.J. Palvimo, O. Silvennoinen, Blood 102 (9) (2003) 3311.

[12] Z.L. Yuan, Y.J. Guan, D. Chatterjee, Y.E. Chin, Science 307 (5707) (2005) 269

[13] W. Komyod, U.M. Bauer, P.C. Heinrich, S. Haan, I. Behrmann, J. Biol. Chem. (2005).

[14] K.A. Mowen, J. Tang, W. Zhu, B.T. Schurter, K. Shuai, H.R. Herschman, M. David, Cell 104 (5) (2001) 731.

[15] T. Meissner, E. Krause, I. Lödige, U. Vinkemeiwer, Cell 119 (5) (2004) 587.

[16] C.C. Lin, C.M. Chou, Y.L. Hsu, J.C. Lien, Y.M. Wang, S.T. Chen, S.C. Tsai, P.W. Hsiao, C.J. Huang, J. Biol. Chem. 279 (5) (2004) 3308.

[17] J. Castelli-Gair Hombría, S. Brown, J. Curr. Biol. 12 (16) (2002) R569.

[18] X.S. Hou, Z. Zheng, X. Chen, N. Perrimon, Dev. Cell 3 (6) (2002) 765.

[19] R. Yan, S. Small, C. Desplan, C.R. Dearolf, J.E. Darnell Jr., Cell 84 (3) (1996) 421.

[20] X.S. Hou, M.B. Melnick, N. Perrimon, Cell 84 (3) (1996) 411.

[21] R. Binari, N. Perrimon, Genes Dev. 8 (3) (1994) 300.

[22] D.A. Harrison, P.E. McCoon, R. Binari, M. Gilman, N. Perrimon, Genes Dev. 12 (20) (1998) 3252.

[23] S. Brown, N. Hu, J. Castelli-Gair Hombría, Curr. Biol. 11 (21) (2001) 1700.

[24] P. Karsten, S. Hader, M.P. Zeidler, Mech. Dev. 117 (1-2) (2002) 343.

[25] J.S. Rawlings, G. Rennebeck, S.M. Harrison, R. Xi, D.A. Harrison, MC Cell Biol. 5 (1) (2004) 38.

[26] A. Betz, N. Lampen, S. Martinek, M.W. Young, J.E. Darnell Jr., Proc. Natl. Acad. Sci. U. S. A. 98 (17) (2001) 9563

[27] H. Luo, P. Rose, D. Barber, W.P. Hanratty, S. Lee, T.M. Roberts, A.D. D’Andrea, C.R. Dearolf, Mol. Cell Biol. 17 (3) (1997) 1562. 
[28] M. Meister, M. Lagueux, Cell Microbiol. 5 (9) (2003) 573.

[29] H. Agaisse, U.M. Petersen, M. Boutros, B. Mathey-Prevot, N. Perrimon, Dev. Cell 5 (3) (2003) 441.

[30] T. Mukherjee, J.C. Hombría, M.P. Zeidler, Oncogene 24 (15) (2005) 2503.

[31] E.A. Bach, N. Perrimon, in: P.B. Sehgal, D.E. Levy, T. Hirano (Eds.), Signal Transducers and Activators of Transcription (STATs); Activation and Biology, Kluwer Academic Publishers, p. 87.

[32] A.H. Brand, N. Perrimon, Development 118 (2) (1993) 401-415.

[33] E.J. Kwon, H.S. Park, Y.S. Kim, E.J. Oh, Y. Nishida, A. Matsukage, M.A. Yoo, M. Yamaguchi, J. Biol. Chem. 275 (26) (2000) 19824.

[34] G.M. Rubin, A.C. Spradling, Science 218 (4570) (1982) 348-353.

[35] G. Halder, P. Callaerts, W.J. Gehring, Science 267 (5205) (1995) 1788.

[36] I. Schneider, J. Embryol. Exp. Morphol. 27 (2) (1972) 353.

[37] R. Lehmann, D. Tautz, in: L.S.B. Goldstein, E.A. Fyrberg (Eds.), Drosophila melanogaster: Practical Uses in Cell and Molecular Biology, Academic Press, p. 576.

[38] K. Ariyoshi, T. Nosaka, K. Yamada, M. Onishi, Y. Oka, A. Miyajima, T. Kitamura, J. Biol. Chem. 275 (32) (2000) 24407.
[39] J. Li, W. Li, H.C. Calhoun, F. Xia, F.B. Gao, W.X. Li, Mech. Dev. 120 (12) (2003) 1455

[40] K.M. McBride, N.C. Reich, in: P.B. Sehgal, D.E. Levy, T. Hirano (Eds.), Signal Transducers and Activators of Transcription (STATs); Activation and Biology, Kluwer Academic Publishers, p. 269.

[41] D.A. Harrison, R. Binari, T.S. Nahreini, M. Gilman, N. Perrimon, EMBO J. 14 (12) (1995) 2857.

[42] S.M. Sweitzer, S. Calvo, M.H. Kraus, D.S. Finbloom, A.C. Larner, J. Biol. Chem. 270 (28) (1995) 16510.

[43] M.P. Zeidler, E.A. Bach, N. Perrimon, Oncogene 19 (21) (2000) 2598.

[44] E.A. Bach, S. Vincent, M.P. Zeidler, N. Perrimon, Genetics 165 (2003) 1149.

[45] Y.C. Tsai, Y.H. Sun, Genesis 39 (2) (2004) 141.

[46] R. Wilk, I. Weizman, B.Z. Shilo, Genes Dev. 10 (1) (1996) 93.

[47] M.A. Henriksen, A. Betz, M.V. Fuccillo, J.E. Darnell, Genes Dev. 16 (18) (2002) 2379.

[48] S. Brown, N. Hu, J. Castelli-Gair Hombría, Development 130 (14) (2003) 3077.

[49] X. Chen, S.W. Oh, Z. Zheng, H.W. Chen, H.H. Shin, S.X. Hou, Dev. Cell 4 (2) (2003) 179 